\title{
Circuit
}

Musiques contemporaines

\section{L’opéra audiovisuel dans le cinéma québécois : vecteur de l'expérimentation sonore}

\section{Réal La Rochelle}

Volume 19, numéro 3, 2009

Pionniers canadiens de la lutherie électronique

URI : https://id.erudit.org/iderudit/038259ar

DOI : https://doi.org/10.7202/038259ar

Aller au sommaire du numéro

Éditeur(s)

Les Presses de l'Université de Montréal

ISSN

1183-1693 (imprimé)

1488-9692 (numérique)

Découvrir la revue

Citer cet article

La Rochelle, R. (2009). L’opéra audiovisuel dans le cinéma québécois : vecteur de l'expérimentation sonore. Circuit, 19(3), 66-76.

https://doi.org/10.7202/038259ar
Résumé de l'article

Le cinéma québécois, depuis les années 1950, a été l'objet d'un assez grand nombre d'expérimentations sonores filmiques. Les principales ont été produites à l'ONF grâce, au départ, aux travaux de Maurice Blackburn, Norman McLaren et Arthur Lipsett. Au fil des décennies, les semences de ce mouvement d'avant-garde ont poussé dans un nombre assez important de films, plusieurs au studio d'animation, d'autres par une intervention significative dans le cinéma direct. À l'heure actuelle, ces filmopéras se pratiquent surtout dans la vidéo d'art et s'inscrivent dans des performances multimédias et des installations muséales. 


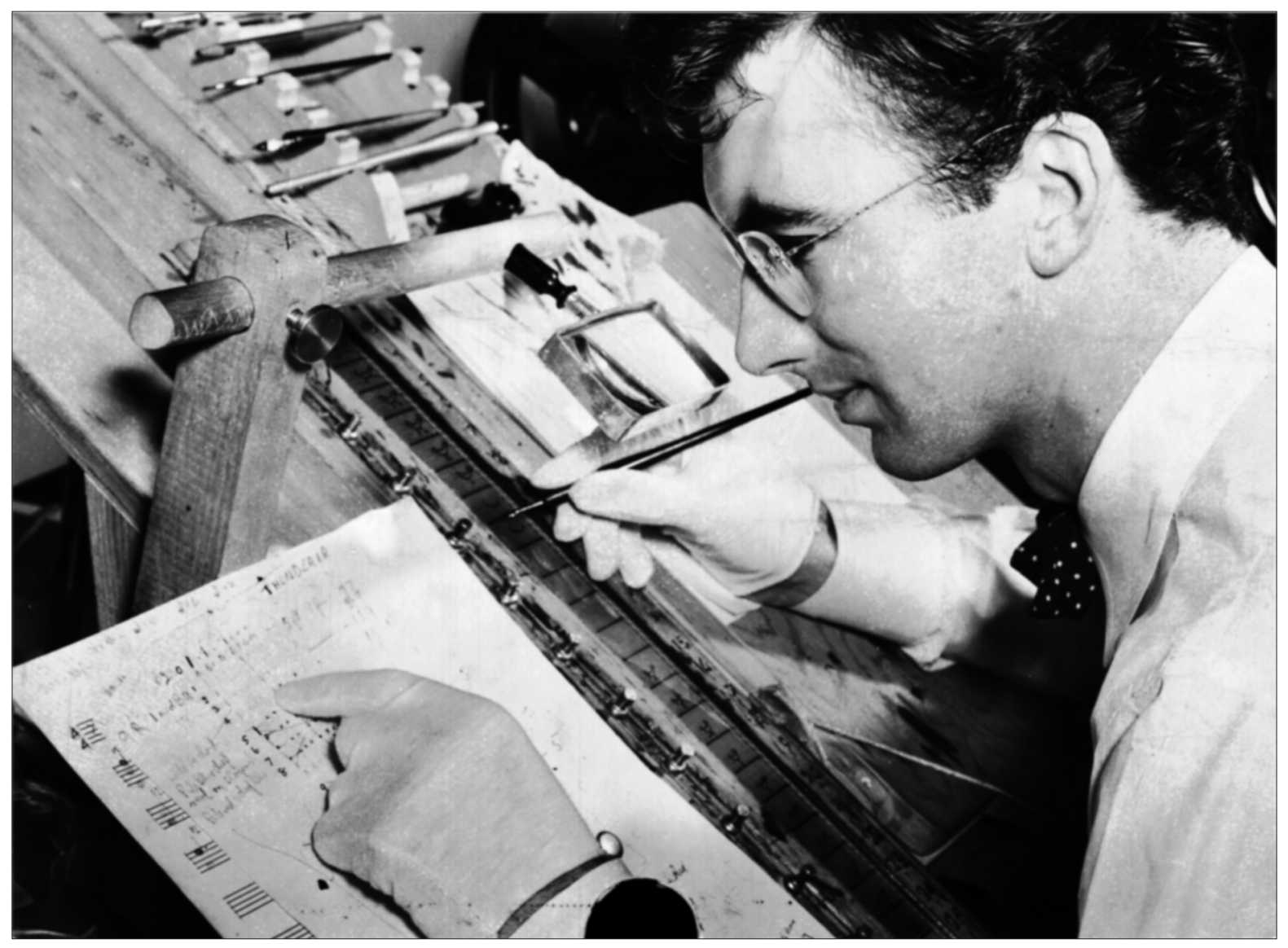

Norman McLaren travaillant à une séquence du film Hen Hop (fait sans caméra, 1942).

Photographie utilisée avec la permission de I'Office national du film du Canada. 


\section{L’opéra audiovisuel dans le cinéma québécois : vecteur de l'expérimentation sonore}

Réal La Rochelle

La lutte historique, incessante et interminable, à l'œuvre dans le sonore filmique, c'est d'abord le combat d'une bande en celluloïd qui veut se faire entendre et se laisser écouter. C'est aussi une tentative de briser les «conventions naturalistes » des synchronismes entre sons et images en faveur d'un contrepoint sonore, «fondement d'une esthétique du nouvel art audiovisuel». Autrement dit, non plus pour illustrer une sorte d'illusion de la vérité du réel, mais pour «faire naître des interrogations en vue de créer de nouvelles entités» (Mari, 2007, p. 15).

Dans Protée, Michel Larouche (1985) a bien illustré les pôles de cette question fondamentale dans les rapports images/sons'. Rappelant que diverses «expériences de jumelage aboutissent toutes à un étonnant parallélisme dans leur incapacité à résoudre les divergences entre ces moyens d'expression indépendants a priori: l'image et le son» (Larouche, 1985, p. 68), l'essayiste montre qu'il n'est pas utopique de viser «le désynchronisme systématique de la musique et des voix, pris comme des éléments d'égale importance dans la constitution du film, libérés de leur traditionnelle fonction illustrative » (Larouche, 1985, p. 68). Prenant appui sur des films de Marguerite Duras, de Werner Schroeter et de Michael Snow, Larouche explore les cas de films expérimentaux où niche la «co-présence d'occurrences visuelles et sonores, autrement dit qu'il y [a] un travail dialectique sur le son, un véritable montage audio-visuel »(Larouche, 1985, p. 69).

Le présent article vise à retracer les moments les plus révélateurs, dans le cinéma québécois, de cette «lutte avec l'ange » du sonore qui veut se mesurer avec le visuel.
1. Plusieurs des films mentionnés dans cet article peuvent être visionnés sur le site de l'Office national du film du Canada à l'adresse <www.onf.ca $>$. 


\section{La symbiose image/son : une utopie?}

À l'Office national du film du Canada (ONF), le compositeur Maurice Blackburn est le premier à mettre de l'avant l'expérimentation sonore filmique et à l'inscrire à la fois dans le cinéma canadien-anglais (en particulier dans les films d'animation de Norman McLaren) et dans le cinéma québécois naissant, dans tous les genres et formats. Blackburn va d'abord explorer ce champ avec Norman McLaren, lequel, avant même son entrée à l'ONF en 1941, avait expérimenté à New York une forme de musique concrète, en dessinant ou en gravant des sons musicaux directement sur pellicule. Allegro (1939), Points et Boucles, en 1940, participent à cet effort créatif, reconduit à l'ONF, en 1952, dans Voisins et Two Bagatelles. Blackburn (1995) propose une sorte de concept englobant pour désigner les films issus de son laboratoire: «C'est peut-être parce que je conçois le cinéma comme un opéra qu'il m’est difficile de penser aux images, aux bruits, au commentaire, à la musique, comme si ces éléments pouvaient être compartimentés, isolés les uns des autres.»

Ce faisant, ce compositeur québécois, qui a travaillé en France avec Pierre Schaeffer et Pierre Henry, vise le «filmopéra » au même titre que la «filmusique » que prône après coup en France Michel Fano, en se basant sur les expérimentations d'Alban Berg dans ses opéras Wozzeck et Lulu. Idée que Jean-Claude Mari (2007, p. 219) désigne par «le modèle opératique bergien ». Cette notion de «filmopéra » est un terme inventé par Kurt Weill (1993), qui le distingue de l'opéra filmé, soit la captation par des caméras d'un spectacle lyrique scénique. À l'opposé, le filmopéra est avant tout l'adaptation cinématographique d'un opéra, donc n'obéissant plus aux lois du théâtre mais à celles du langage filmique. Par exemple, La Flûte enchantée de Bergman, ou encore le Parsifal de Syberberg sont des filmopéras, alors que moult représentations du Metropolitan ou de La Scala sont des opéras filmés. Cette matrice bergienne, à l'œuvre dans ces nouvelles expérimentations audiovisuelles, traverse plusieurs œuvres cinématographiques québécoises qui émergent de ce chantier exploratoire.

Une fois ce socle posé, une fois ainsi déclenchée la bataille contre le faux naturalisme filmique, cette expérimentation sonore opératique rencontre un dilemme majeur: synchronisme ou contrepoint? Car ce n'est pas tout d'ouvrir la bande sonore filmique à une nouvelle résonance (le travail sur les voix, les bruitages, le «sound design», les musiques), encore faut-il la situer dans une dynamique qui «participe au renouvellement de la nature du lien qui unit les composantes sonores et leurs fonctions vis-à-vis de l'image, du récit». Comment arriver «à l'édification d'une nouvelle esthétique sonore, musicale»? (Mari, 2007, p. 19-20). 
Tiraillement techno-esthétique à l'œuvre aussi dans le cinéma québécois. Le travail musical de Maurice Blackburn en la matière est révélateur de cette tension. Tout en reconnaissant à ce compositeur d'avoir « un véritable projet sonore » et en notant "qu'il est très étonnant d'avoir pensé le son de cette manière dans les années 1950 et 1960» (Blackburn, 1995), Michel Fano fait remarquer que les trames musicales de Blackburn pour les films de McLaren, A Phantasy (1952) et Blinkity Blank (1955), sont redevables d'une volonté de synchronisme, ce qui est d'ailleurs la norme pour les musiques de tous les films de McLaren. C'est aussi le cas du travail sonore de Blackburn avec Bretislav Pojar sur le film E (1981), une partition éblouissante, mais enclavée dans l'ordre de la synchronicité.

En revanche, quand Blackburn obtient de certains réalisateurs entière liberté pour composer des bandes sonores, par exemple pour Je (1961) de Louis Portugais, ou encore pour Jour après jour (1962) de Clément Perron, alors il crée des valeurs inédites de contrepoint entre l'image et le son. Dans ces deux cas, il s'agit de véritables filmopéras modernes, comme en rêvait déjà Kurt Weill en 1930, quand il envisageait le «film-opéra futur» appuyé sur «les possibilités musicales et les lois formelles fondamentales du cinéma sonore» (Kurt Weill 1993, p. 87). Le documentaire-essai Jour après jour demeure sans doute l'épitomé du filmopéra datant des débuts du cinéma québécois, surtout qu'il est produit au moment où s'imposent le direct synchrone et cette curieuse notion de "cinéma de la parole», dans le sens d'une relation vive entre l'image du locuteur et le son de sa voix. Bien au contraire, le film de Perron et Blackburn revendique une complète autonomie de la bande image (scènes de type «réaliste » montrant des ouvriers au labeur) et de la trame sonore, leur unique rapport dialectique étant celui d'une réflexion poétique et musicale sur la machine moderne qui façonne l'homme à son image.

Un long poème, lu par Anne Claire Poirier, est entrelacé de musiques concrètes et de divers bruitages de galopades de chevaux, de trains, de sifflets, d'arbres qui s'écroulent. Maurice Blackburn avait en tête de monter à l'ONF un atelier sonore, voué à la conception et à la réalisation sonore. Un projet alors assez mal compris, qui n’empêchait pas d'autres cinéastes, en dehors de ce rayon d'action, de s'adonner à quelques bricolages sonores. Au sein du nouveau cinéma québécois direct, où prime le culte du synchronisme image/ son, ces expérimentations fragmentées paraissent assez surprenantes, encore que parfois difficilement décelables.

Marcel Carrière, ingénieur du son, met ainsi en lumière la « fausse synchro » à l'œuvre dans Les Raquetteurs (1958) et des séquences du Mardi gras 
dans Pour la suite du monde (1963), films considérés pourtant comme fondateurs du direct synchrone. Gilles Groulx, de son côté, introduit dans Le Chat dans le sac (1963) des sound designs de coups de feu (symboles d'une éventuelle révolution armée au Québec), qui n’ont rien de réaliste. Ce cinéaste a par ailleurs influencé Denys Arcand qui, dans ses premiers courts métrages historiques, fabrique ses montages sonores comme des tapisseries de voix, de musiques et de bruitages en contrepoint avec les images. Ainsi résonne la trilogie Champlain (1964), Les Montréalistes et La Route de l'Ouest (1965).

Arthur Lipsett joue aussi des mêmes techniques pour Very Nice Very Nice (1961) et 21-87 (1962). En 1961 encore, Claude Jutra invente un commentaire sonore étonnant pour le collectif La Lutte, en faisant alterner des sons d'ambiance tonitruants d'aréna en délire et des musiques de Bach. Dans Bûcherons de la Manouane, Arthur Lamothe offre à Blackburn les traitements fantasmatiques de coups de hache dans les forêts glacées. Tous ces exemples, bien écoutés, révèlent une autre facette de la lutte avec l'ange.

Pendant les années 1970, à l'Atelier de conception et de réalisations sonores, enfin mis sur pied par Blackburn, le compositeur électroacousticien Yves Daoust accomplit une percée remarquable de filmusique, à caractère opératique moderne, sur deux films de Jacques Leduc. Pour Samedi: le ventre de la nuit (1977) ainsi que Le Plan sentimental (1978), dernier épisode et épilogue de la suite Chronique de la vie quotidienne, Daoust développe, en contrepoint avec les images et les sons live du cinéaste, des partitions de bruitisme musical d'une grande beauté. Le compositeur refuse les trames formées des seuls sons produits par la machine, l'électroacoustique pur, pour traiter plutôt les sons cueillis par le réalisateur (bruitages, bribes de paroles et de chansons) et les inscrire dans une toile tissée par l'ordinateur. Et ce, toujours en contrepoint, rarement en synchronisme, sauf quand l'exigent quelques séquences de cinéma prises sur le vif. De la sorte, Daoust intègre à merveille les données sonores du direct avec la manipulation électronique, une symbiose qui magnifie musicalement les vertus brutes de la prise de vue synchrone. Riche ensemble où dominent les crachements des micros et des haut-parleurs, le bric-à-brac strident de la cabine de projection d'un ciné-parc, les radios et les magnétophones des voisins; une brève plage au ralenti ponctue la fin du marathon de danse, un passage vif de clavecin électronique après le bingo; à la fin du film, sur les images de démolition du cinéma Capitol, s'élève la chanson populaire «Grand-maman, oh! oui grand-maman, vous avez dû passer par là... » Mais ces créations d'Yves Daoust sont des exemples dispersés, dans l'underground de l'ONF, presque confidentiels. 
Entre les années 1950 et 1970, s'est donc implantée à l'ONF l'idée d'un travail spécifique à développer en regard de la bande sonore filmique. Toutefois, à cause de mille tracasseries administratives, à cause aussi de la résistance de plusieurs cinéastes qui ne souhaitaient pas s'éloigner du synchronisme images/sons du cinéma direct, le projet d'établir en permanence un atelier du son est resté fragile, puis a fini par s'effondrer.

Mais la graine était semée et c'est paradoxalement durant les années 1980 que les plus belles fleurs du travail sonore vont éclore. Durant cette décennie, vont apparaître des films de Bretislav Pojar, Gilles Groulx, Fernand Bélanger et Pierre Hébert, des compositeurs comme Blackburn, Jacques Hétu, René Lussier, Robert Marcel Lepage et Jean Derome, des concepteurs sonores comme Claude Beaugrand et Catherine Van Der Donckt qui, tous, donnent au cinéma québécois l'une de ses plus belles moissons de filmopéras.

\section{Filmopéras onéfiens des années 1980}

Quand le cinéaste Bretislav Pojar réalise son film E (1981), sarcasme dévastateur des totalitarismes, il bénéficie d'une création sonore de Maurice Blackburn, une de ses plus achevées, en dépit du fait qu'elle fonctionne en synchronisme avec l'image animée. Le compositeur fait de l'opéra la parodie d'un art bourgeois, dictatorial, élitiste, qu'il confie à un chœur joyeux et débridé. Par ailleurs, pour ses bruitages, Blackburn fait appel, de manière originale, au duo Les Mimes électriques, qui produisent avec la bouche des bruits ressemblants à des sons réalistes mais qui ont une petite allure dérangeante, presque fantasmatique.

A la même période, le dernier long métrage de Gilles Groulx, Au pays de Zom, se présente comme un ovni et laisse bouche bée l'administration de l'ONF. La dernière séquence, en particulier, accomplit un métissage singulier et adroit entre le filmopéra et le cinéma direct. Dans un premier temps, Monsieur Zom (interprété par la basse chantante Joseph Rouleau) clame son faux repentir d'homme riche au moyen d'un texte parlé-chanté (les italiens l'appellent, en opéra, le parlar cantando) qui, petit à petit, s'épanouit en chant. Zom, qui se rêve en chanteur d'opéra, déclare vouloir mourir sur une scène. Raccord à la mort du tyran dans le Boris Godounov de Moussorgski. Cette scène terminée, la caméra fait un zoom arrière, montrant le public dans un amphithéâtre, qui se lève et se disperse après le spectacle. Ce dernier fragment est ponctué de bruitages synchrones de murmures, de fauteuils qui craquent, de bruissements des spectateurs. Ce film est un véritable filmopéra postmoderne, dont la musique est de Jacques Hétu. Si ce long métrage 
2. Ndlr: En 2007, de nombreuses œuvres de Pierre Hébert, parmi lesquelles La Plante humaine, Chants et danses du monde inanimé - Le Métro, Adieu bipède et La Lettre d'amour, ont été regroupées par l'ONF dans le coffret «Pierre Hébert - La science des images animées/The Science of Moving Images" [ONF 183C 9907 050], qui contient 3 DVD (19 films en versions française et anglaise) et 1 CD sur lequel est reproduite la bande sonore du film Explosion démographique/Population Explosion interprétée par The Ornette Coleman Trio. fonctionne lui aussi dans l'ordre de la synchronicité, son effet est néanmoins percutant, et il demeure à ce jour une rare incursion d'un filmopéra dans le long métrage québécois. Il brille toujours de ses mille feux audacieux, même si sa circulation reste plutôt confidentielle.

Mieux publicisés, les films-spectacles que déploie Pierre Hébert pendant une dizaine d'années font preuve d'une originalité assez hilarante, quoique au fond très grave. C'est avec Etienne et Sara (1984) que le cinéaste d'animation démarre sa collaboration avec les compositeurs de musique actuelle René Lussier, Robert Marcel Lepage et Jean Derome. Par la suite, Hébert décide de se lancer dans des performances où il s'adonne à la gravure en direct sur des boucles de films $16 \mathrm{~mm}$, alors que les musiciens improvisent leurs compositions jazziques. Après coup, le cinéaste utilise ces matériaux pour en faire des films. Ainsi vont Chants et danses du monde inanimé - Le Métro (1985), Ô Picasso (tableaux d'une surexposition) (1985), Adieu bipède (1987), La Lettre d'amour (1988)². Dans tous ces courts métrages, s'installe l'autonomie respective des musiques et des images animées pour la construction de la symbiose filmique.

Passiflora (1985), de Fernand Bélanger et Dagmar Gueissaz-Teufel, perdure comme un des sommets du filmopéra à l'ONF. Basé sur les visites à Montréal, très médiatisées, de Michael Jackson et de Jean-Paul II, ce long métrage déconstruit ces événements, les coule dans des contre-propositions combatives et anarchiques, souvent désynchronisées, au moyen de commentaires sonores créés à la fois par les musiciens René Lussier, Jean Derome et André Duchesne et le concepteur sonore Claude Beaugrand. Il en résulte un patchwork éblouissant et très contrôlé, une mosaïque d'images et de sons qui demeure un chef-d'œuvre du cinéma québécois. Par exemple, les paroles du pape au Stade olympique, "prosternez-vous », sont ironiquement musicalisées par Lussier et entrelacées aux sons live traités en décor sonore par Beaugrand. La trame sonore participe de la sorte à la fictionnalisation du reportage, à la mise en essai critique du compte rendu d'événements médiatiques, nourrit le second degré de ce qui s'appelle le documentaire, participe à la destruction de ce faux genre.

\section{Des aventures sonores filmées}

En empruntant cette expression à Fernand Bélanger pour définir son long métrage Le Trésor archange (1996), il est possible de caractériser la période d'expérimentations sonores des années 1990 jusqu'à aujourd'hui. Une époque qui voit d'un côté le démantèlement de la production à l'ONF, donc la fin des avant-gardes filmiques (sauf quelques rares cas en animation), de l'autre 
l'implantation massive de la vidéo et de l'électronique tous azimuts, qui permet le montage des studios privés et la réalisation chez soi. Il n'empêche. Plusieurs œuvres, urbi et orbi, témoignent d'explorations sonores parmi les plus fines que le cinéma québécois ait produites depuis ses origines, il y a un demi-siècle.

Le studio d'animation de l'ONF voit l'éclosion de certains films majeurs comme La Plante humaine de Pierre Hébert (1996) et Le Chapeau de Michèle Cournoyer (1999). Le studio offre également l'aide à la postproduction des filmopéras Aria (2001) de Pjotr Sapegin et Ce que dit la bouche d'ombre (2004) de Jean-François Jung.

Pour son dernier film à l'ONF avant sa retraite, Hébert s'attaque à un long métrage, le seul de sa carrière. Mélangeant l'animation et la prise de vue réelle (elle-même tantôt brute, tantôt métissée d'animation), La Plante humaine est une magnifique déambulation, contemporaine et diachronique, dans le monde des images, des contes mythiques, un questionnement poétique et rutilant du sens de la communication et de la mémoire. Sur le plan sonore, la musique de Robert Marcel Lepage et les décors sonores de Claude Beaugrand s'entrelacent aux images avec empathie et distanciation, ce qu'accomplit de manière semblable la très belle composition de Jean Derome pour Le Chapeau, percutante animation de Michèle Cournoyer portant sur les viols d'enfants.

Pour Aria, Normand Roger signe une des très belles partitions de sa carrière de musicien de films, un très émouvant condensé de quelques minutes de l'opéra Madama Butterfly de Puccini. Jean-François Jung réalise Ce que dit la bouche d'ombre avec ces poèmes de Victor Hugo qui disent «tous les gouffres souterrains, sous-marins ou galactiques », qui murmurent aussi les «tombes et les sépulcres » grâce aux voix et aux bouches des tables parlantes. Le cinéaste nous conduit au cœur même d'une des formes modernes de l'art lyrique, espace audiovisuel de l'entrecroisement des bruitages inquiétants, des voix d'outre-tombe, des musiques des «chaudrons bouillants» (Jung, 2004), où s'inscrivent le hiératisme des gestes et des postures des comédiens ainsi que les décors fantastiques des dessins hugoliens. Tout concourt dans ce film à construire une scène audiovisuelle pour le filmopéra, lieu de prédilection pour le renouveau contemporain de l'art lyrique.

Ailleurs, le long métrage de Fernand Bélanger, Le Trésor archange, s'arrime au singulier Trésor de la langue de René Lussier, une composition inusitée qui fut d'abord une pièce radiophonique, puis un disque, ensuite une œuvre pour le concert. Ici, paroles musicalisées, décors sonores de Claude Beaugrand, montage images et sons par le réalisateur servent à ce dernier à 
faire émerger une œuvre singulière, autre gloire de l'expérimentation sonore filmique.

Sinon, d'autres œuvres marquantes s'expriment par la vidéographie. Rien ne t'aura, mon coeur (1997), de Charles Guilbert et Serge Murphy, est un musical dans lequel le minimalisme des personnages, des objets, des décors et des musiques tisse le plus étrange des filmopéras de cette fin de siècle. À noter également le fascinant La Voisine (1995) de Diane Poitras, ballet-théâtre qui emprunte au mime, au cabaret et au stand-up comic, avec Dulcinée Langfelder, sur des musiques de Christian Calon et de Lepage. Ce dernier signe aussi la trame sonore de Du front tout autour de la tête (2000) de Chantal duPont, un vidéogramme poignant sur le cancer. Un autre exemple intéressant de cette période est L'Invention d'un paysage (1998), vidéogramme de Serge Cardinal, Martin Allard et Louis Comtois (réalisateur, preneur de son et concepteur sonore). Film né du «désir de saisir par le sonore un effet de solitude et de mélancolie inscrit dans le paysage; le désir d'injecter un peu d'étrangeté dans la réalité matérielle par la musicalisation des bruits et la bruitisation de la musique » (Cardinal, 2002, p. 162). Enfin, comment ne pas évoquer cette autre vidéo hors norme de Manon Labrecque, Actions (2007), extraite de l'installation Exercices de neutralité, qui capte l'image mais surtout les sons du très original platiniste Martin Tétreault, dont les jeux avec les disques et la lutherie phonographique forment un ensemble halluciné et très riche. La question centrale, dans l'imbroglio entre le direct synchrone et l'expérimentation sonore, demeure celle de la permanence et de la pertinence d'une idée du cinéma direct québécois comme «cinéma de la parole». À l'ONF, cette idée a été comprise comme l'ultime expression d'un «cinéma vérité », vite devenu un dogme, rapidement un mythe. Un diktat qui mettait toute autre démarche comme une lampe sous le boisseau. D'abord, en restreignant le son filmique au verbe dominant, mettant ainsi en arrière-plan les éléments de bruitages, de musiques et de décors sonores. Ensuite, en décrétant que la seule véracité de ce verbe était sa synchronicité avec l'image.

Dans cette optique, une autre voie, une vision différente, se trouvaient dès lors suspectes. Une bataille à multiples visages s'enclenchait: administrative, politique, esthétique. Une sorte de lutte entre pragmatiques et rêveurs, entre réalistes et utopistes. Un combat où la paresse du conformisme - la facilité relative de la synchronicité - faisait reculer la complexité et le long temps de travail de recherches en acoustique, en musique contemporaine, en créativité bruitiste. Le flux abondant du «cinéma de la parole» refoulait l'expérimentation sonore dans les marges et les limbes. 
Or, ce qu'ont prôné moult résistants est que le cinéma direct est compatible avec le filmopéra. Bien plus, que le filmopéra peut être l'expression la plus achevée du direct hissé au niveau de l'art, plutôt que pataugeant dans la répétition ennuyeuse du synchronisme sonore, ce qui a conduit, jusqu'à plus soif, aux interminables films de chaises ou de cuisines. Il n'était déjà pas facile de faire croire à la vérité réaliste de l'image, alors que les choix des sujets et des intervenants, les cadrages, les mouvements de caméra et le montage contredisent les prétentions à la crudité du réel; il n'était pas non plus aisé de laisser entendre que les sons étaient de leur côté à ce point fidèles à la nature qu'ils pouvaient faire oublier comment les microphones sont des passeurs et opèrent des métamorphoses, que le travail sur ces sons en direct existe en studio, que la bande sonore est le résultat d'un montage et d'un mixage d'une grande complexité techno-esthétique.

Une certaine notion du cinéma direct a conduit à faire prendre des vessies pour des lanternes, ou encore comme on nomme «assurance-vie » ce qui n'est qu'une assurance sur la mort. Que tant de producteurs et de réalisateurs aient cru à ce leurre, que tant de critiques et d'analystes leur aient emboîté le pas, montre bien qu'un certain conformisme et une paresse intellectuelle cimentent l'idée d'un réel, illusoire, plutôt que de laisser le champ libre à l'entière illusion contrôlée, assumée, de l'art cinématographique.

Le filmopéra, dans l'expérimentation sonore du cinéma québécois, magnifie bien davantage le direct que les nombreux tours de magie du cinéma vérité.

En guise de coda, il est intéressant d'écouter/voir l'un des meilleurs exemples de ces dernières années, celui des vidéogrammes de Pierre Hébert. Filmopéras, non dans le sens strict et premier d'œuvres lyriques pour le cinéma, comme le sont Au pays de Zom et Aria, mais dans l'acception plus large, métaphorique, avancée par Michel Fano et Maurice Blackburn, le film dans sa totalité audiovisuelle comme opéra, comme «modèle opératique bergien » (Mari, 2007, p. 219).

Hors les murs de l'ONF, Pierre Hébert continue sa production de films singuliers, particulièrement avec le musicien californien Bob Ostertag. Ils produisent en duo de captivantes performances vidéomusicales, dont le cinéaste tire ensuite des films en solo: Between Science and Garbage (2002), Variations sur deux photographies de Tina Modotti (2004), La Technologie des larmes (2005). Nonobstant leurs prouesses techniques, ces deux auteurs de filmopéras choisissent des sujets à forte connotation idéologico-politique. Ils s'insurgent contre l'impérialisme américain, l'administration Bush et les 
guerres au Proche-Orient, se révoltent en montrant un monde au bord du gouffre apocalyptique.

La Statue de Giordano Bruno (2005) ramène à la surface contemporaine le spectre de l'Inquisition et de sa barbarie organisée. Un seul plan-séquence forme tout le film, qui montre la statue de Bruno sur une place de Rome. En surimpression, des flammes enveloppent la statue et tout ce qui bouge autour, autos et passants. Ce bûcher, avec ses mille lueurs de rouge et de sang, avec sa trame sonore crépitante, devient l'élément destructeur qui anéantit l'image, sème la mort de la philosophie et de la pensée libre, de l'humain hors norme, du cinéma même qui en véhicule l'expression. Un filmopéra qui, à sa manière, est un "crépuscule des dieux» et de l'homme, l'issue d'un combat de l'humain contre les fausses idoles et les pouvoirs de violence et de mort.

Métaphore de la lutte avec l'ange, ce film porte en lui la symbolique d'un cinéma expérimental dans lequel sons et images se dressent contre tous les conformismes et travaillent à réconcilier l'ouïe et la vue. L'issue de ce corps à corps est incertaine - ce peut être la mort - mais seule compte, en fin de nuit, la valeur de cette lutte, qu'une aube nouvelle, comme la lumière d'un écran, vient illuminer.

\section{B I B L I O G RA P H IE}

Maurice Blackburn. Filmusique. Filmopéra (1995), livret du coffret phonographique Analekta (AN 2 7005-6), Montréal, La Phonothèque québécoise/Musée du son.

Cardinal, Serge, Allard, Martin et ComTOis, Louis (2002), «La musicalité d'une bande sonore. À propos de L'Invention d'un paysage», dans Écouter le cinéma, Montréal, Les 400 coups, coll. «Cinéma », p. 158-174.

JunG, Jean-François (2004), ciné-fiche de présentation du film Ce que dit la bouche d'ombre, Montréal, ONF.

LAROUCHE, Michel (1985), «Vers la symbiose image-son dans le cinéma expérimental: une utopie?», Protée, vol. 13, n² 2, p. 67-71.

MARI, Jean-Claude (2007), Quand le film se fait musique. Une nouvelle ère sonore au cinéma, Paris, L'Harmattan.

WeILL, Kurt (1993), «Film sonore, opéra filmé, film-opéra », HuYnH Pascal (dir.), Kurt Weill de Berlin à Broadway, Paris, Plume, p. 82-88. 